\title{
The ResQCPR System: An Objective and Systematic Review
}

\author{
Glenn Goodwin, D.O., EMT-P, RN-BSN ${ }^{1,2}$, Tarik Zeid, D.O. ${ }^{1}$, Todd Belok, D.O. ${ }^{1}$ \\ ${ }^{1}$ Touro College of Osteopathic Medicine, Department of Primary Care, 230 W 125th St., New York, NY 10027 \\ ${ }^{2}$ Aventura Hospital and Medical Center, Emergency Department, 20900 Biscayne Blvd., Miami, FL 33180
}

*Corresponding Author: Glenn Goodwin, D.O.; ggoodwin954@gmail.com

Received 17 November 2021;

Accepted 27 November 2021;

Published 01 December 2021

\begin{abstract}
The resource-limiting environment of the pre-hospital setting necessitates the continuous development of tools and interventions that maximise the capabilities of emergency medical services (EMS) municipalities. One such product developed by Zoll, the ResQCPR system, attempts to enhance the effectiveness of prehospital cardiopulmonary resuscitation (CPR). The ResQCPR system is comprised of the ResQPOD, which is an impedance threshold device (ITD), and the ResQPUMP, which is an assisted compression-decompression (ACD) device. Limited data exists regarding Zoll's specific apparatuses as well as similar such devices. This systematic analysis comprehensively summarises a number of related studies. Their methods, limitations, results, and other aspects are outlined in the respective sections. Conclusion: This review concludes by determining that the effectiveness of the ResQPUMP, in particular (and ACD devices in general), is promising while the ResQPOD's is uncertain. Future studies are needed to determine whether the effectiveness of the ResQPUMP markedly diminishes when used independently of the ResQPOD. This is an important feature for fire departments and other EMS municipalities because outfitting all appropriate units with both devices can be quite costly. Further studies are also needed to demonstrate reproducibility in humans (because 2 of the studies used pigs as test subjects) and with a greater amount of test subjects.
\end{abstract}

Kevwords: Assisted Compression Device; Impedance Threshold Device; ResQCPR; ResQPOD; ResQPUMP

\section{Introduction}

Cardiac arrest is one of the most common emergencies seen in the pre-hospital setting. There are over 326,000 out of hospital cardiac arrest episodes per year and only $8.3 \%$ of which have adequate return of neurological function following the episode [1]. Expeditious restoration of adequate blood flow to vital organs, primarily cerebral blood, flow is imperative. Paramedics and other health care personnel accomplish this by performing procedures outlined in basic life support (BLS) and advanced cardiac life support (ACLS), which commonly involve maneuvers of chest compression providing the basis of cardiopulmonary resuscitation (CPR). Manual compressions in these procedures, however, may not always be performed optimally due to the emergent nature of the situation or other various physiological barriers ${ }^{[2]}$. Quality chest compressions and airway establishment, when used in conjunction with defibrillation, remain as the most effective determinants of positive patient outcome ${ }^{[3-7]}$

Consequently, many products have been introduced that attempt to maximise the effectiveness of compressions and ventilations. The focus of this review will be on Zoll's ResQCPR System. Limited studies exist in addressing the effectiveness of these products but there is still enough to draw reasonable conclusions.

\section{Methods and Materials}

A search of the National Library of Medicine's MEDLINE/PubMed as well as Google Scholar was performed with the goal of finding all articles published in English language with "ResQCPR" or "ResQPOD ResQPUMP" in the title. We mainly selected recent publications, but did not exclude older works that were widely referenced. All data were accessed between May and July 2017. Our comprehensive PubMed/Medline search revealed a total of over 100 manuscripts that mentioned an aspect of the ResQCPR system but only four studies were pertinent to this review.

\section{The ResQCPR System}

The ResQCPR system is comprised of two apparatuses: the ResQPOD and the ResQPUMP, aimed at maximising the effectiveness of cardiopulmonary resuscitative efforts. By forcefully pulling the chest cavity upwards, greater negative intrathoracic pressure is achieved leading to greater filling into the heart and therefore more blood flow upon compression. The ResQPOD is a small device attached to the endotracheal tube that acts as a one-way valve in preventing air from entering back into the chest cavity upon chest recoil. This also attempts to achieve greater negative intrathoracic pressure. The device manufacturer 
claims more effective compressions with greater reproducibility, inherent ease of use, and a $49 \%$ increase in survival, up to one year ${ }^{[8]}$. The results of the studies included in this article indicate that the ResQPUMP is remarkably effective while the ResQPOD is indeterminate. Studies have suggested the benefit of use of such devices, however, consensus must be reached on their effectiveness. Herein we aim to analyse the various studies that have been conducted to objectively discuss the utilisation of the ResQCPR system. The figures below demonstrate the effects of the ResQCPR system.

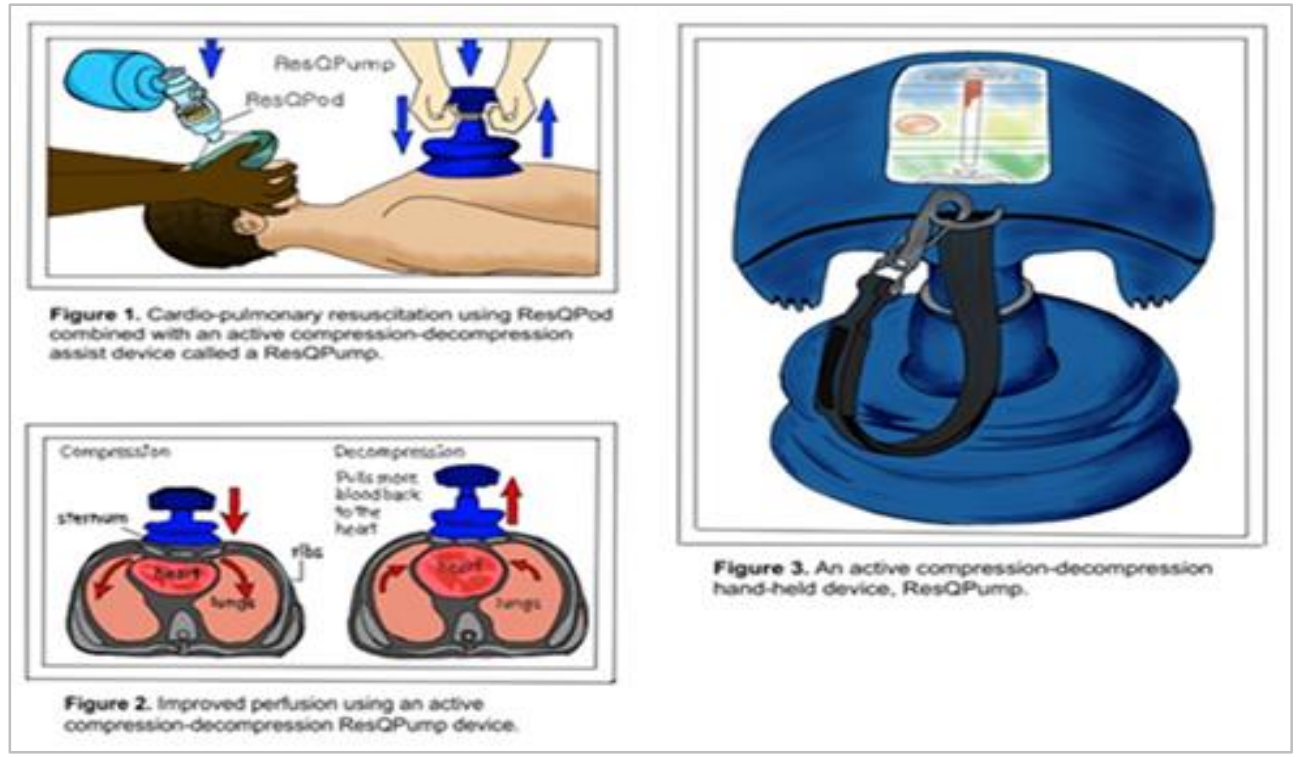

Figure 1: Above figures recreated by Jeffrey Nguyen, D.O., EMT-B, and extracted from the Lobodzinski study ${ }^{[9]}$.

\section{Discussion}

Resuscitation assist devices have been gradually introduced over the past decades to help optimise patient outcomes. As cardiac arrest remains one of the more common emergent pre-hospital scenarios, utilisation of these devices should be wide spread ${ }^{[10,11]}$. The goals of such devices are to maximise compressions and ventilations because those are two of the most important factors correlating to positive patient outcomes in cardiac arrest ${ }^{[12]}$. Optimising ventilations and compressions is essentially the goal in every cardiac arrest encounter and the ResQCPR system addresses both of those. The studies have been separated based on their physiological or clinical focus.

\section{Physiologically Focused}

To accurately assess the utilisation of the ResQCPR device, individual components must be reviewed. Performing CPR involves a compression component where the chest wall is compressed to simulate cardiac systolic function. A great barrier to this action may be chest wall compliance ${ }^{[13,14]}$. Chest wall compliance is essentially the difference in pressure between the chest cavity and atmospheric pressure. If chest compliance is increased, greater intrathoracic volumes can be achieved, thereby exploiting the Starling Effect; greater intrathoracic volume leads to increased ventricular filling, and subsequent increased end organ perfusion ${ }^{[15,16]}$. Studies have shown that CPR assist devices, like the ResQPUMP, improved chest compliance which improves end organ blood flow ${ }^{[17-19]}$. One study concluded that chest compliance is increased significantly using the ResQPUMP ${ }^{[20]}$. To identify potential changes in chest compliance, anterior posterior (AP) chest height measurements were performed on newly deceased (never frozen) human cadavers during CPR. 2 separate measurements were taken: one before and another one after 5 minutes of automated CPR ${ }^{[20]}$. Segal et al. tested the hypothesis that "after 5 minutes of CPR chest compliance would be significantly increased by applying static compression (30, 40, and $50 \mathrm{~kg})$ and decompression forces $(-10,-15 \mathrm{~kg})$ with the ResQPUMP". Automated CPR was done for five minutes and it was determined that the same force compressed the chest significantly more than before (the use of the ResQPUMP) ${ }^{[20]}$. The active decompression significantly contributed to this subsequent ease of compressions. Lateral chest $\mathrm{x}$-rays were obtained with multiple reference markers to assess changes in AP distance. The Segal study's use of actual intrathoracic photographs was beneficial because it provides direct visual evidence supporting their findings. Graphical and quantitative representation of the aforementioned is provided below.

Table 1: Segal et al. Chest Compliance Table: Anterior Posterior (AP) Chest Dimensions during Active Compression and Decompression CPR. The Segal et al. Chest Compliance Table reflects the change in AP chest height before and after 5 minutes of automated C-CPR. The AP excursion distances, after the 5 minutes of automated CPR, were significantly greater during each of the compressions and decompressions measured. One of the cadavers had the sternum break during the first compression and decompression measurements. Another cadaver had the ribs broken during the static compression at $40 \mathrm{~kg}$ after the $5 \mathrm{minutes}$ of automated CPR ${ }^{[20]}$.

\begin{tabular}{|l|l|l|}
\hline Force & Decrease in AP (in cm) without CPR & Decrease in AP after 5 mins of automated CPR (in cm) \\
\hline Compression $30 \mathrm{~kg}$ & 2.1 & 3.7 \\
\hline Compression $40 \mathrm{~kg}$ & 2.9 & 4.9 \\
\hline Compression $50 \mathrm{~kg}$ & 4.3 & 6.3 \\
\hline Decompression $-10 \mathrm{~kg}$ & 1 & 2.3 \\
\hline Decompression $-15 \mathrm{~kg}$ & 1.8 & 2.7 \\
\hline
\end{tabular}




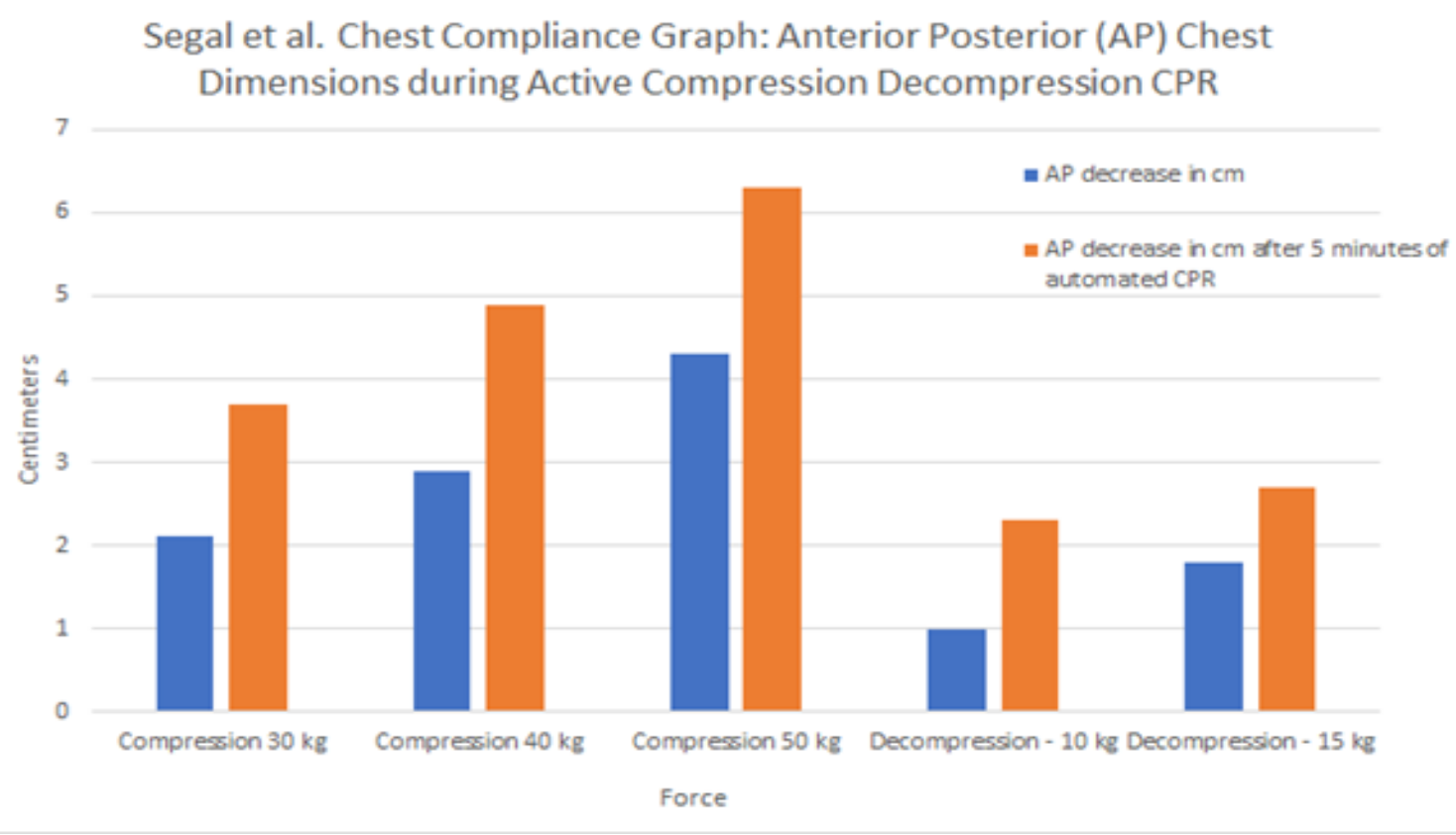

Figure 2: Segal et al. Chest Compliance Graph reflects the data from the Segal et al. Chest Compliance Table. The AP decrease was greater for each of the compression and decompression measurements made after 5 minutes of automated CPR20.

Determining the success of these CPR devices is also done by considering the neurological outcomes of test subjects. While achieving a return of spontaneous circulation in subjects is an indicator of an effective device, it's important to determine whether the brain parenchyma is receiving adequate perfusion. The brain is particularly susceptible to hypoxic damage because it lacks regenerative capabilities found in most other organs ${ }^{[21]}$. Metzger et al.'s study addressed this by the following: "Seventeen pigs were subjected to 8.5 minutes of untreated ventricular fibrillation and prospectively randomized to cardiopulmonary resuscitation at 80 chest compressions/min or active compression-decompression cardiopulmonary resuscitation at 80 chest compressions/min plus an impedance threshold device. Coronary perfusion pressures, carotid blood flow, and 24-hr neurological survival were higher with active compression-decompression cardiopulmonary resuscitation + an impedance threshold device ${ }^{[22]}$. Cerebral perfusion pressures, measured in nine additional pigs, were improved with active compression-decompression cardiopulmonary resuscitation + an impedance threshold device. With active compression-decompression cardiopulmonary resuscitation + impedance threshold device, mean diastolic intracranial pressure during decompression was lower and the downward slope of the decompression phase intracranial pressure curve was steeper". It was concluded that active compressiondecompression cardiopulmonary resuscitation plus an impedance threshold device increased cerebral perfusion pressures while simultaneously lowering diastolic intracranial pressure during the decompression phase. Quantitative representation of the data can be found in tables and figures below. These mechanisms were proposed by the authors to explain the observed increases in cerebral perfusion pressure, carotid blood flow, and survival rates, with the additional favorable neurologic outcomes. The practical limitation of this study is that the focus was only up to 24-hours post-resuscitation. Other studies that explored neurological outcomes in post cardiac arrest patients followed them up to a year after their cardiac arrest episode ${ }^{[23]}$. Many patients changed significantly (improved or declined) from their initial 24-hour assessment. The limited time frame of this study inhibits one's ability to draw more comprehensive conclusions concerning neurological outcomes. With this limitation in mind, however, cerebral perfusion was still increased in the active compressiondecompression + ITD group compared to the conventional compression group.

Table 2: Metzger et al. 24-hour Survival Table: "24-hour Survival Assessment of CPR at 80 CC/min vs. ACD CPR + ITD. 24-hour Survival Table reflects the 24-hour survival data with favorable neurological function. Both methods of CPR utilized 80 chest compressions per minute. The survival outcomes were significantly worse for the nine pigs in the CPR at $80 \mathrm{CC} / \mathrm{min}$ when compared to the eight pigs treated with the ACD CPR + ITD method. One pig in the ACD CPR + ITD group was excluded from this analysis due to the incorrect calibration of the lead cell which measures compression forces. 24-hour survival with good neurological function was measured by utilizing cerebral performance score of 1 or 2 (CPC 1 or 2), which is based on a 5-point scoring system with $1=$ normal and 5 = brain death" ${ }^{[22]}$.

\begin{tabular}{|l|l|l|}
\hline 24-hour assessment & $\begin{array}{l}\text { CPR at 80 Chest Compressions/min } \\
(\mathbf{n = 9})\end{array}$ & $\begin{array}{l}\text { Active Compression Decompression CPR + Impedance } \\
\text { Threshold Device (APC CPR + ITD) (n=8) }\end{array}$ \\
\hline 24-hour survival & 4 & 8 \\
\hline 24-hour survival with CPC 1 or 2 & 2 & 7 \\
\hline
\end{tabular}




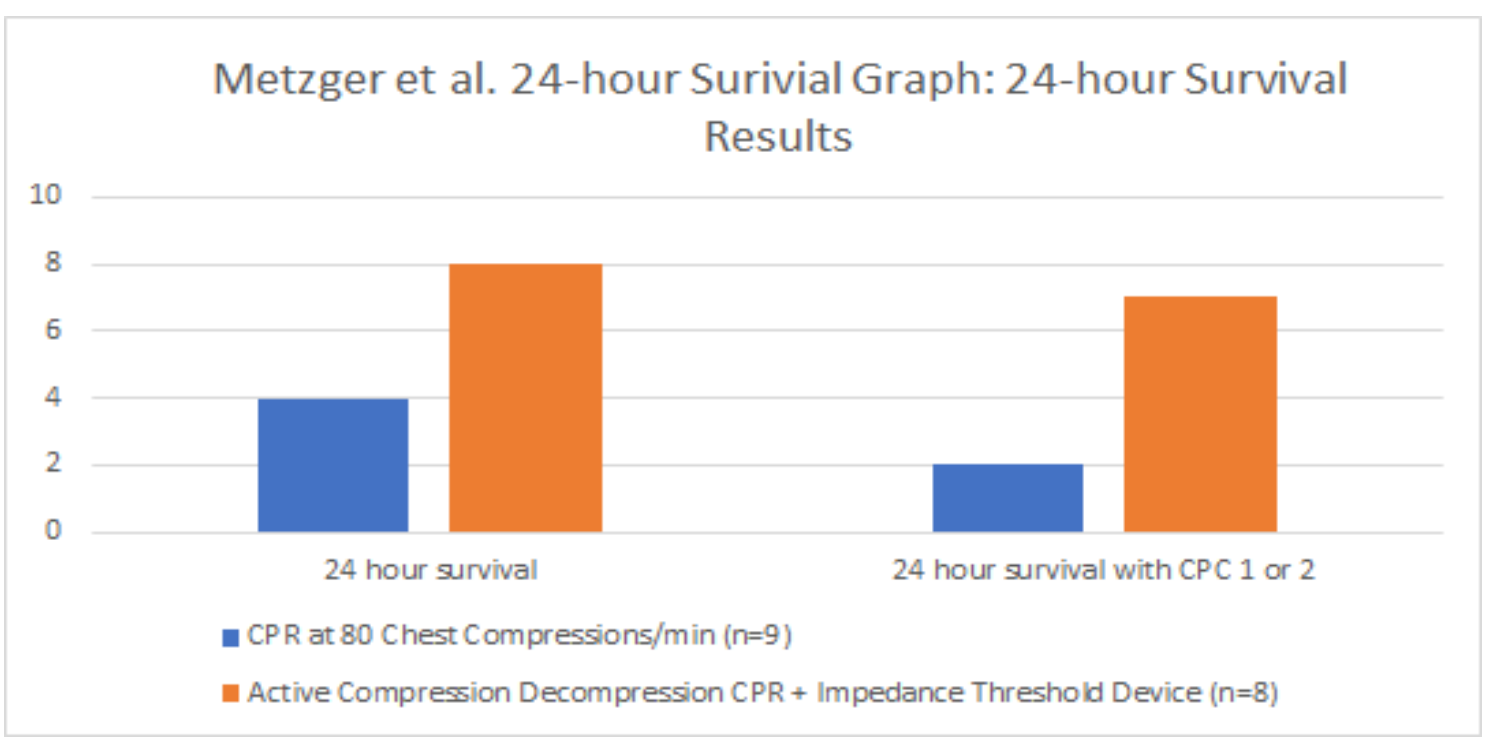

Figure 3: Metzger et al. 24-hour Survival Graph reflects that data from the corresponding 24-hour Survival Table. "The 24-hour survival and 24-hour survival with CPC 1 or 2 were significantly better for the group that received ACD CPR + ITD when compared to the group that had CPR at $80 \mathrm{CC} /$ minute. There were 9 pigs in the CPR at $80 \mathrm{CC} /$ minute group and 8 pigs in the ACD CPR + ITD group" ${ }^{222]}$.

Table 3: Metzger et al. Neurological Assessment Table: Neurological Assessment of ACD CPR + ITD and CPR at 80 CC/minute. Neurological Assessment Table represents that neurological assessment score with the mean score as well as the standard deviation. These results were collected after 8.5 minutes of ventricular fibrillation and six minutes of either methodology of CPR. The results between the two groups were not statistically significant due to insufficient data because only four pigs survived the $80 \mathrm{CC} / \mathrm{minute}$ group and only two of those four pigs had neurological deficits that were measurable. In all categories considered, all but one of the categories showed that the $80 \mathrm{CC} /$ minute had greater neurological deficits than the ACD CPR + ITD group ${ }^{[22]}$.

\begin{tabular}{|l|l|l|l|l|}
\hline $\begin{array}{l}\text { Neurological Assessment } \\
\text { Swine Neurological } \\
\text { Deficit Score) }\end{array}$ & $\begin{array}{l}\text { CPR at 80 Chest } \\
\text { Compressions/min (n=9) }\end{array}$ & $\begin{array}{l}\text { Standard } \\
\text { Deviation (+/-) }\end{array}$ & $\begin{array}{l}\text { Active Compression Decompression } \\
\text { CPR + Impedance Threshold } \\
\text { Device (n=8) }\end{array}$ & $\begin{array}{l}\text { Standard } \\
\text { Deviation (+/-)2 }\end{array}$ \\
\hline Consciousness & 30 & 17.3 & 15 & 5.7 \\
\hline Respiratory Pattern & 0 & 0 & 0 & 0 \\
\hline Painful Stimulus & 18.8 & 12 & 4.4 & 3.2 \\
\hline Muscle Tone & 25 & 14.4 & 3.1 & 3.1 \\
\hline Standing & 10 & 5.8 & 2.5 & 2.5 \\
\hline Walking & 15 & 8.7 & 5 & 3.8 \\
\hline Restraint & 25 & 14.4 & 7.5 & 5.3 \\
\hline Total Deficit Score & 124 & 72 & 33 & 17 \\
\hline
\end{tabular}

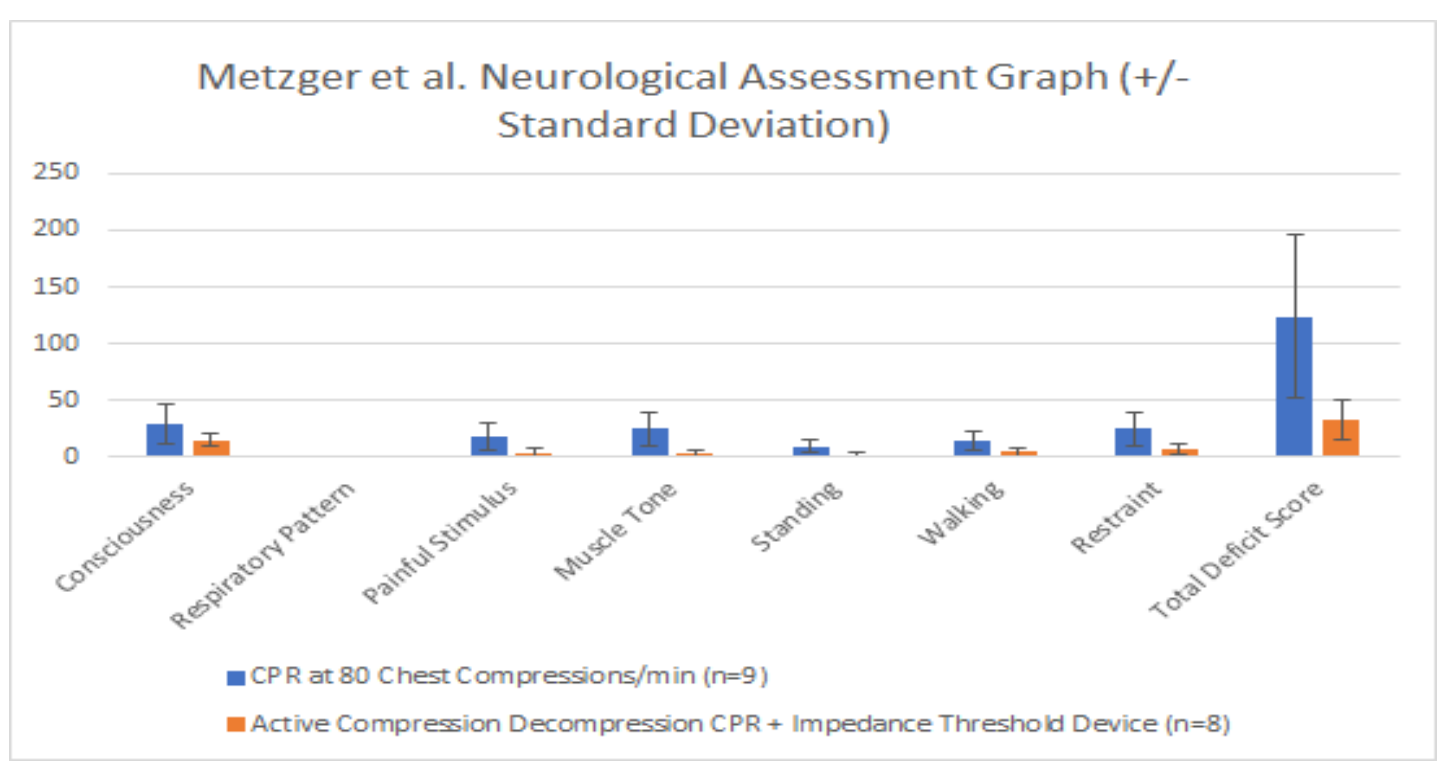

Figure 4: Metzger et al. Neurological Assessment Graph is a representation of the corresponding Neurological Assessment Table that shows that the $80 \mathrm{CC} /$ minute group had greater neurological deficits than the ACD CPR + ITD group. The only category in which the two groups had a similar neurological deficit score was the respiratory pattern. The neurological deficits measured by way of the Swine Neurological Deficit Score. The total deficit score was much greater for the 80 CC/minute group than the ACD CPR + ITD group ${ }^{[22]}$. 


\section{Clinically Focused}

The practical benefits elicited from the Segal study is exemplified by another study which compared patient outcomes in patients receiving CPR against patients receiving CPR+ResQCPR system. While many patients obtain a return of spontaneous circulation (a spontaneous heart beat) following a cardiac arrest, the lack of oxygen to the brain and other organs results in permanent and debilitating damage ${ }^{[21]}$. To assess outcomes in a more comprehensive manner, researchers studied survival rates in addition to neurological functioning in patients that received care with the ResQCPR apparatus ${ }^{[9]}$. It was concluded that survival rates can be improved by up to $50 \%$ when using the two devices together. The Lobodizinski study utilized seven EMS municipalities throughout the US, involving 46 organizations in urban, suburban, and rural areas. 2,470 patients were randomized, with 1,653 qualifying for the examination. 840 patients were in the intervention group, in which ResQCPR interventions (ResQPod and ResQPump) were administered with CPR, while 813 patients received standard CPR without ResQCPR apparatuses. Their outcomes were tracked throughout their hospital stay and their resultant neurological outcomes were compared using the Rankin scoring system. Lobodzinski's study included jurisdictions spanning a wide range of areas and demographics which made it highly randomised and dynamic. This study did a great job of accounting for the myriad of environments in which cardiac arrest episodes take place, however, the elimination criteria to select the 1,653 patients out of 2,470 patients was never mentioned. To elaborate, studies of this nature typically eliminate subjects due to their arrest being traumatic in nature or other factors such as age, medical history, and down time ${ }^{[24]}$ (the time elapsed between a patient being in cardiac arrest and receiving CPR). Because we don't know which patients were included, it's difficult to draw definitive conclusions because many other factors may have contributed to unfavorable or favorable outcomes, completely independent from the ResQCPR apparatus. With this limitation in mind, however, there was still a remarkable increase in survivability in patients receiving CPR with the ResQCPR system to those who didn't.

Table 4: Lobodzinski ResQCPR versus Conventional CPR Outcome Comparison Table: Discharge with favorable neurological function, survival and adverse event rates, cardio-pulmonary resuscitation (CPR) with ResQ interventions vs standard CPR

\begin{tabular}{|l|l|l|l|}
\hline & CPR with ResQ interventions (n= 840) & Standard CPR $(\mathbf{n}=\mathbf{8 1 3})$ & P \\
\hline Discharge with favorable neurological function (\%) & 9 & 6 & 0.019 \\
\hline Survival to discharge (\%) & 12 & 10 & 0.12 \\
\hline Survival at one year (\%) & 9 & 6 & 0.03 \\
\hline No major adverse event (\%) & 6 & 6 & 0.68 \\
\hline Pulmonary edema (\%) & 11 & 8 & 0.015 \\
\hline
\end{tabular}

\section{Lobodzinski ResQCPR versus Conventional CPR Outcome Comparison Graph: Discharge with favorable neurological function, survival and adverse event rates, cardio-pulmonary resusictation (CPR) with ResQ interventions vs standard CPR}

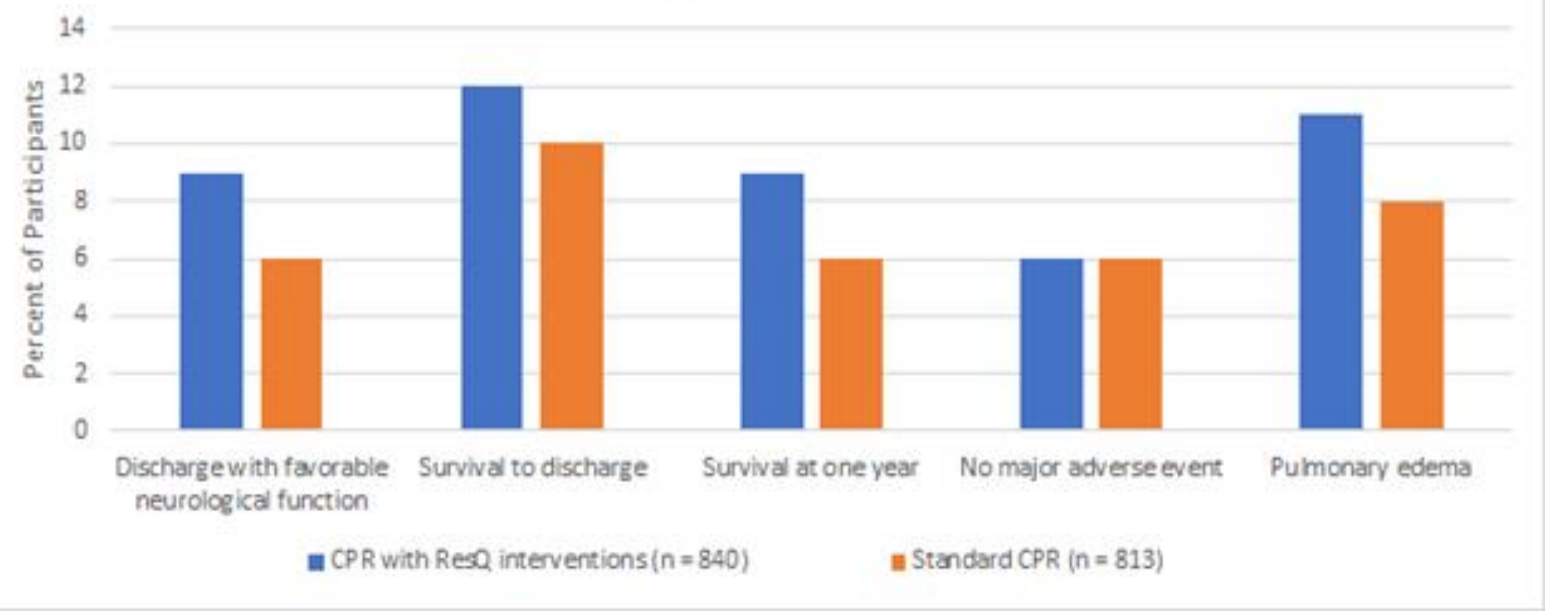

Figure 5: Lobodzinski ResQCPR versus Conventional CPR Outcome Comparison Graph represents the "percentages of favorable neurological function, survival and adverse event rates, CPR with ResQ interventions vs standard CPR of 1653 patients with 813 in the control group and 840 in the intervention group. The ResQ intervention group had a greater percentage of patients with discharge with favorable neurological function, survival to discharge, survival at one year and pulmonary edema" ${ }^{\text {[9] }}$

While the ResQPUMP's effectiveness has been evidenced, the ResQPOD's is less conclusive ${ }^{[25]}$. The objective of the ResQPOD is to increase negative intrathoracic pressure, resulting in improved systemic blood circulation ${ }^{[8]}$. Achieving greater negative intrathoracic pressure allows for increased ventricular filling and subsequent increase in blood and perfusion to end organs ${ }^{[26]}$. A study conducted by Johnson et al. attempted to determine if the
ResQPOD does in fact improve systemic circulation. 12 pigs were placed in two groups: CPR with the ResQPod® and CPR without the use of the ResQPod ${ }^{\circ}$. Cardiac arrest was achieved in the pigs by IV potassium chloride injection. After two minutes of CPR, epinephrine was administered by IV push injection and then serum samples were collected at various time intervals up to 10 minutes after the injection. Results are reported in means and standard 
deviations respectively. The two parameters that were studied was time to maximum drug plasma concentration (Tmax) and maximum plasma concentration (Cmax), which are regarded as standard pharmacokinetic principles underlying intravenous drug absorption and distribution ${ }^{[27]}$. Tmax indicates the time needed for a drug to reach desired or effective absorption within a margin of few minutes. A delay may come about from the time it takes the drug to reach the particular sampling site ${ }^{[27]}$. "Use of the ResQPod® resulted in lower Cmax than control. Tmax was longer when using ResQPod® compared to the control group. Although there were differences between the groups, the results were not statistically significant relative to Cmax and Tmax ( $p=0.276)$ " [28]. A graphical and table representation of this can be found further down in this section. The theoretical foundation of that study is that the time to maximum epinephrine concentration levels should be decreased in the ResQPOD group due to the increased venous return to the heart ${ }^{[28]}$. However, the results of this study and another study by Jenkins et al. suggest that there is no significant difference between drug concentrations with and without use of the ResQPOD ${ }^{[28,29]}$.

Table 5: Johnson et al. Tmax and Cmax Comparison Study of Conventional Intervention versus ResQPod Intervention: Time to Maximum Concentration (Tmax) and Maximum Concentration (Cmax) of Plasma Epinephrine with and without ResQPod

\begin{tabular}{|l|l|l|l|}
\hline Group & Mean & Standard Deviation $(+/-)$ & N \\
\hline Cmax wih ResQPod & 219.34 & 110.59 & 6 \\
\hline Cmax without ResQPod & 471.53 & 349.71 & 6 \\
\hline Tmax in minutes with ResQPod & 4.75 & 1.54 & 6 \\
\hline Tmax in minutes without ResQPod & 3.42 & 1.11 & 6 \\
\hline
\end{tabular}

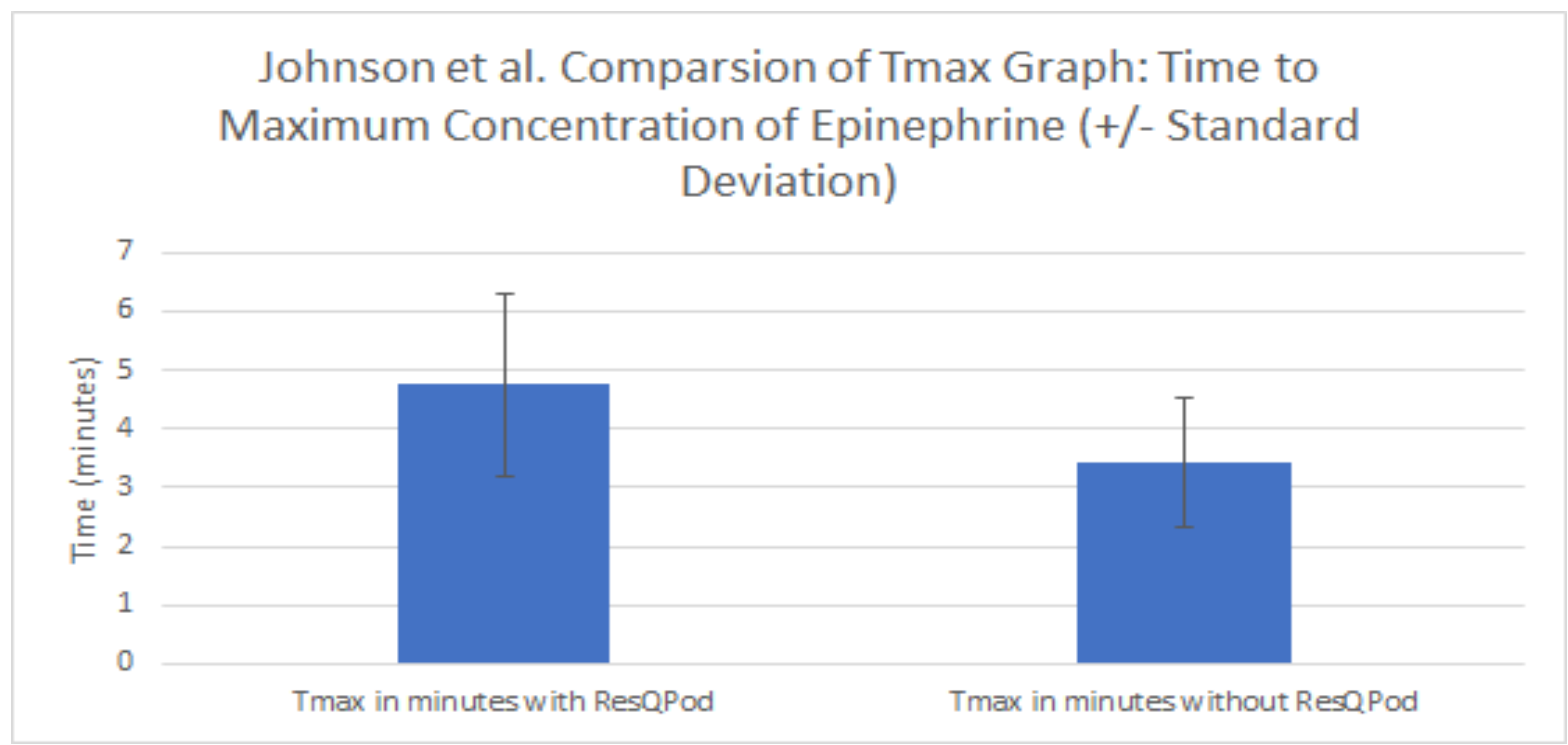

Figure 6: Johnson et al. Comparison of Tmax Graph reflects the Tmax of epinephrine between groups with and without the ResQPod. The standard deviation is represented with the standard error bars. The Tmax was greater in the group with the ResQPod when compared to the control group that did not utilise the ResQPod. Tmax was measured in minutes ${ }^{[28]}$.

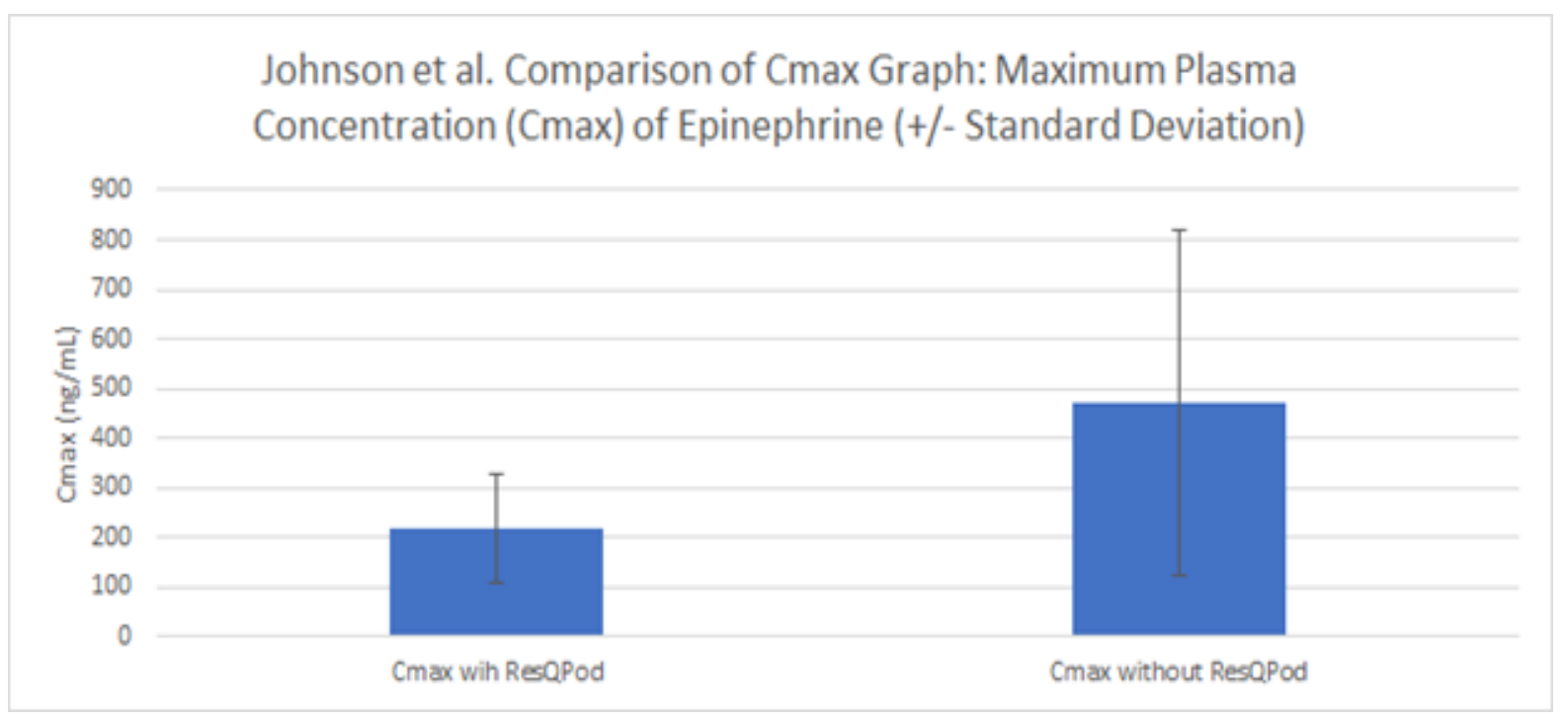

Figure 7: Johnson et al. Comparison of Cmax Graph reflects the Cmax of epinephrine between groups with and without the ResQPod. The Standard deviation is represented with the standard error bars. The Cmax was greater in the control group without the ResQPod when compared to the group that utilised the ResQPod. Cmax is measured in nanograms of epinephrine per milliliter (ng/mL) of plasma ${ }^{[28]}$. 
While the studies had their limitations, they also utilised techniques and practices that produced legitimate results. The Metzger and Johnson studies both employed the use of pigs, which can be advantageous. Pigs are very similar to humans in terms of both their physiology and relative organ proportions ${ }^{[30]}$. They have a few features that make them ideal models for research specifically focused on of cardiac arrest and CPR, many of which are outlined in a study conducted by Cherry et al., 2015, and are provided in the following. Pigs have a large chest cavity, making them suitable for forceful chest compressions (mimicking chest compressions on humans) ${ }^{[31]}$. Serum chemistries, resting heart rate, and blood pressure are very similar between humans and pigs ${ }^{[31,32]}$. There are also neurological examinations that were developed specifically for pigs, which allows researchers to analyse neurological outcomes in a standardised and systematic way ${ }^{[33,34]}$. Their relatively large size facilitates physiological monitoring because some of the monitoring instruments can also be quite large, of which, instruments measuring various parameters such as intra-cardiac and intravascular pressures, electrocardiography, intravenous medications as well as experimental treatments are a few examples. Their large size also results in a large blood volume, allowing researchers to collect many different samples without physiologically altering the pig's health status (or experimental results). A study conducted by Hannon established standard physiologic values for porcine models, and similarities in hematologic properties, pertinent to the conclusions made in other studies, serves to validate the studies even further ${ }^{[35]}$.

\section{Conclusion}

The studies indicate that the ResQPUMP's ability to improve CPR is remarkably effective while the ResQPOD's is ambiguous. While many additional studies are needed to draw a more definitive conclusion, it's safe to expect that the conjunctive use of both devices will significantly improve patient outcomes. Because some of the studies did not explicitly state that they used Zoll's ResQCPR system, it cannot be determined with precision if their ACD and ITD devices significantly alter patient outcomes. The ResQPUMP and ResQPOD may have modifications that improve or worsen their effectiveness as compared to the devices used in the other studies.

\section{Recommendations}

More human-based studies are needed to both, substantiate the effectiveness of the ResQCPR system as well as to determine the unambiguous need for the ResQPOD to be used with the ResQPUMP. While there are not many studies indicating a remarkable effect of using the ResQPOD (solely), none have demonstrated any negative effects. Future studies would probably fall in line with any applicable ethical and moral dilemmas. In addition, more studies are needed to compare the effectiveness between Zoll's ResQCPR system and other similar devices.

\section{Conflict of Interest}

None

\section{Financial Disclosure}

None

\section{References}

[1] Mozaffarian D, Benjamin EJ, Go AS, et al. Heart Disease and Stroke Statistics-2015 Update. A Report from the American Heart Association. 2014.

[2] Wu J-Y, Li C-S, Liu Z-X, Wu C-J, Zhang G-C. A comparison of 2 types of chest compressions in a porcine model of cardiac arrest. The American Journal of Emergency Medicine. 2009;27(7):823-829.

[3] Christenson J, Andrusiek D, Everson-Stewart S, et al. Chest Compression Fraction Determines Survival in Patients With Out-of-Hospital Ventricular Fibrillation. Circulation. 2009;120(13):1241-1247.

[4] Idris AH, Guffey D, Pepe PE, et al. Chest compression rates and survival following out-of-hospital cardiac arrest. Crit Care Med. 2015;43(4):840-848.

[5] University of Arizona SHRRC. Frequently Asked Questions about Chest-Compression-Only CPR. 10/19/13; http://heart.arizona.edu/frequently-askedquestions. Accessed 10/18/17, 2017.

[6] Ristagno G, Tang W, Chang Y-T, et al. The quality of chest compressions during cardiopulmonary resuscitation overrides importance of timing of defibrillation. CHEST Journal. 2007;132(1):70-75.

[7] Lukas R-P, Gräsner JT, Seewald S, et al. Chest compression quality management and return of spontaneous circulation: a matched-pair registry study. Resuscitation. 2012;83(10):1212-1218.

[8] Corporation ZM. ResQCPR System Product Information. 2017; https://www.zoll.com/medicalproducts/impedance-threshold-device/resqcpr/. Accessed 10/18/17, 2017.

[9] Lobodzinski SS. Combined active compressiondecompression cardiopulmonary resuscitation and inspiratory impedance threshold devices may improve survival after out-of-hospital cardiac arrest. Cardiol J. 2011;18(2):207-209.

[10] Aygün M, Yaman HE, Genç A, Karadağl1 F, Eren NB. Mechanical Chest Compression Devices: Historical Evolution, Classification and Current Practices, A Short Review. 2016.

[11] Rubin R. "ResQCPR" Could Help Prevent Cardiac Arrest Deaths. Jama. 2015;313(15):1507-1507.

[12] Corporation ZM. ResQCPR system Frequently Asked Questions. 2016; https://www.zoll.com/-/media/publicsite/products/resqcpr/resqcpr_faqs_final_8-116.ashx?la=en\&hash=BA43DF675ABA6B75072602DA 393348D894F70179. Accessed 10/18/17, 2017.

[13] Weinberg G, O'Connor M. Focus on Physiology to Improve Cardiopulmonary Resuscitation. In: LWW; 2016.

[14] Lurie KG, Nemergut EC, Yannopoulos D, Sweeney M. The physiology of cardiopulmonary resuscitation. Anesthesia \& Analgesia. 2016;122(3):767-783.

[15] Yannopoulos D, McKnite S, Aufderheide TP, et al. Effects of incomplete chest wall decompression during cardiopulmonary resuscitation on coronary and cerebral perfusion pressures in a porcine model of cardiac arrest. Resuscitation. 2005;64(3):363-372.

[16] Costanzo LS. Physiology. 2011; 146-147.

[17] Barash et al. Clinical Anesthesia: Sixth Edition. Philadelphia, PA: Lippincott, Williams, and Wilkins; 2009.

[18] Debaty G, Metzger A, Lurie K. Evaluation of Zoll Medical's ResQCPR System for cardiopulmonary 
resuscitation. Expert review of medical devices. 2015;12(5):505-516.

[19] Wolcke BB, Mauer DK, Schoefmann MF, et al. Comparison of Standard Cardiopulmonary Resuscitation Versus the Combination of Active CompressionDecompression Cardiopulmonary Resuscitation and an Inspiratory Impedance Threshold Device for Out-ofHospital Cardiac Arrest. Circulation. 2003;108(18):22012205.

[20] Segal N, Robinson AE, Berger PS, et al. Chest compliance is altered by static compression and decompression as revealed by changes in anteroposterior chest height during CPR using the ResQPUMP in a human cadaver model. Resuscitation. 2017;116:56-59.

[21] Kumar V, Abbas A, Aster J. Robbins and Cotran Pathologic Basis of Disease. Elsevier; 2015.

[22] Metzger AK, Herman M, McKnite S, Tang W, Yannopoulos D. Improved cerebral perfusion pressures and 24-hr neurological survival in a porcine model of cardiac arrest with active compression-decompression cardiopulmonary resuscitation and augmentation of negative intrathoracic pressure. Crit Care Med. 2012;40(6):1851-1856.

[23] Frascone RJ, Wayne MA, Swor RA, et al. Treatment of non-traumatic out-of-hospital cardiac arrest with active compression decompression cardiopulmonary resuscitation plus an impedance threshold device. Resuscitation. 2013;84(9):1214-1222.

[24] Definition D. Concise Dictionary of Modern Medicine. 2002; $\quad$ https://medicaldictionary.thefreedictionary.com/downtime. Accessed 10/18/17, 2017.

[25] Aufderheide TP, Nichol G, Rea TD, et al. A Trial of an Impedance Threshold Device in Out-of-Hospital Cardiac Arrest. New England Journal of Medicine. 2011;365(9):798-806.

[26] Klabunde R. Factors Promoting Venous Return. 2008; http://www.cvphysiology.com/Cardiac\%20Function/CF0 18. Accessed 10/18/17, 2017.

[27] Reijers JAA, Dane MJC, van Zonneveld AJ, Burggraaf J, Moerland M. Potential Influence of Endothelial Adsorption on the Delayed Time to Maximum Concentration of Biopharmaceuticals. European Journal of Drug Metabolism and Pharmacokinetics. 2017.

[28] Johnson D, Rushton J, Hernandez M, et al. Effects of the ResQPod ( ${ }^{\circledR}$ on Maximum Concentration and Time to Maximum Concentration of Epinephrine in a Porcine
Cardiac Arrest Model. Analgesia \& Resuscitation: Current Research. 2013;2(0).

[29] Jenkins C, Brinkley K, Alford H, et al. Effects of the ResQPOD on Kinetics, Hemodynamics of Vasopressin, and Survivability in a Porcine Cardiac Arrest Model. Mil Med. 2015;180(9):1011-1016.

[30] Walters EM, Agca Y, Ganjam V, Evans T. Animal models got you puzzled?: think pig. Annals of the New York Academy of Sciences. 2011;1245(1):63-64.

[31] Cherry BH, Nguyen AQ, Hollrah RA, Olivencia-Yurvati AH, Mallet RT. Modeling cardiac arrest and resuscitation in the domestic pig. World J Crit Care Med. 2015;4(1):1-12.

[32] Swindle MM, Makin A, Herron AJ, F. J. Clubb J, Frazier KS. Swine as Models in Biomedical Research and Toxicology Testing. Veterinary Pathology. 2012;49(2):344-356.

[33] Sullivan S, Friess SH, Ralston J, et al. Improved behavior, motor, and cognition assessments in neonatal piglets. Journal of neurotrauma. 2013;30(20):1770-1779.

[34] Eisenberg MM. Cardiac Arrest Survival Information. 2017. Accessed 10/24/17, 2017.

[35] Hannon JP, Bossone CA, Wade CE. Normal physiological values for conscious pigs used in biomedical research. LETTERMAN ARMY INST OF RESEARCH PRESIDIO OF SAN FRANCISCO CA;1989.

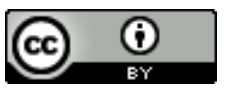

Open Access This article is licensed under a Creative Commons Attribution 4.0 International License, which permits use, sharing, adaptation, distribution and reproduction in any medium or format, as long as you give appropriate credit to the original author(s) and the source, provide a link to the Creative Commons license, and indicate if changes were made. The images or other third party material in this article are included in the article's Creative Commons license, unless indicated otherwise in a credit line to the material. If material is not included in the article's Creative Commons license and your intended use is not permitted by statutory regulation or exceeds the permitted use, you will need to obtain permission directly from the copyright holder. To view a copy of this license, visit https://creativecommons.org/licenses/by/4.0/.

(C) The Author(s) 2021 